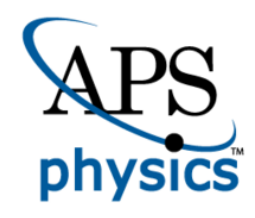

This is the accepted manuscript made available via CHORUS. The article has been published as:

\title{
Magnetopolaritons in Weyl Semimetals in a Strong Magnetic Field
}

Zhongqu Long, Yongrui Wang, Maria Erukhimova, Mikhail Tokman, and Alexey Belyanin Phys. Rev. Lett. 120, 037403 - Published 18 January 2018 DOI: 10.1103/PhysRevLett.120.037403 


\title{
Magneto-polaritons in Weyl semimetals in a strong magnetic field
}

\author{
Zhongqu Long, ${ }^{1}$ Yongrui Wang, ${ }^{1}$ Maria Erukhimova, ${ }^{2}$ Mikhail Tokman, ${ }^{2}$ and Alexey Belyanin ${ }^{1}$ \\ ${ }^{1}$ Department of Physics and Astronomy, Texas AछMM University, College Station, TX, 77843 USA \\ ${ }^{2}$ Institute of Applied Physics, Russian Academy of Sciences
}

(Dated: December 14, 2017)

\begin{abstract}
Exotic topological and transport properties of Weyl semimetals generated a lot of excitement in the condensed matter community. Here we show that Weyl semimetals in a strong magnetic field are highly unusual optical materials. The propagation of electromagnetic waves is affected by an interplay between plasmonic response of chiral Weyl fermions and extreme anisotropy induced by a magnetic field. The resulting magneto-polaritons possess a number of peculiar properties, such as hyperbolic dispersion, photonic stop bands, coupling-induced transparency, and broadband polarization conversion. These effects can be used for optical spectroscopy of these materials including detection of the chiral anomaly, or for broadband terahertz/infrared applications.
\end{abstract}

PACS numbers:

Weyl semimetals (WSMs) have unusual electronic and transport properties originating from the nontrivial topology of the Brillouin zone [1-3]. They have been studied experimentally, mostly with angle-resolved photoemission spectroscopy; e.g. [4-6]. The most intensely studied phenomena include topologically protected surface states known as Fermi arcs, the chiral anomaly, or the non-conservation of the chiral charge in parallel electric and magnetic fields, and the resulting anomalous magnetoresistance [7-10]. Optics of WSMs received relatively less attention so far. Far-infrared optical spectroscopy studies of TaAs without the magnetic field have been recently reported [11]. The conductivity, magnetoplasmons, and polaritons in a magnetic field were calculated recently in quasiclassical approximation [12-18]. The strong-field optical conductivity was calculated in [19]. Here we concentrate on the wave propagation in WSMs in a strong magnetic field, when the electron motion transverse to the field becomes quantized. We show that hybridization of magnetoplasmons with electromagnetic (EM) waves in WSMs leads to fascinating optical phenomena involving magnetopolaritons: hyperbolic dispersion, the absence of Landau damping for strongly localized excitations, photonic stop bands, coupling-induced transparency, efficient polarization conversion, and pulse compression, to name a few. We show that optical spectroscopic techniques provide a straightforward and "clean" way of detecting topological properties of low-energy electron states and in particular the chiral anomaly. Moreover, WSMs show strong promise for future photonic chips enabling a wide array of broadband optoelectronic applications, such as polarizers, modulators, switches, and pulse shapers for mid-infrared through terahertz wavelengths.

Dielectric tensor for a WSM in a magnetic field We consider the material which has only one pair of Weyl nodes for simplicity, with low-energy excitations around each node described by the Weyl Hamiltonian,

$$
H=\chi \hbar v_{F} \boldsymbol{\sigma} \boldsymbol{k} .
$$

Here $\chi= \pm 1$ is the chirality index, $\boldsymbol{\sigma}$ is a $3 \mathrm{D}$ vector of Pauli matrices, $\boldsymbol{k}$ is the 3D quasimomentum of electrons counted from the Weyl node, and we assumed an isotropic electron dispersion (scalar constant $v_{F}$ ). In a strong magnetic field oriented along $z$ axis the $3 \mathrm{D}$ conical spectrum of quasiparticles near each node is split into Landau-level (LL) subbands $W_{n}$ labeled by the quantum number $n$ :

$$
\begin{aligned}
W_{n} & =\operatorname{sgn}(n) \hbar v_{F} \sqrt{\frac{2|n|}{l_{b}^{2}}+k_{z}^{2}} \text { for } n \neq 0, \\
W_{0}^{(\chi)} & =-\chi \hbar v_{F} k_{z}
\end{aligned}
$$

where $l_{b}=\sqrt{\frac{\hbar c}{e B}}$ is the magnetic length. The electron wavefunctions are given in [20]. We assume that the field is strong enough so that $W_{1}-W_{0}$ at $k_{z}=0$ is much larger than the LL broadening determined by disorder.

The salient feature of the electron spectrum is the presence of chiral electron states with $1 \mathrm{D}$ linear dispersion at $n=0$ LL. The $n=0$ electrons near each node are able to move only in one direction, depending on the sign of $\chi$ and neglecting internode scattering. The majority of peculiar optical properties of WSMs originates from the response of these electron states and its interplay with inter-LL transitions.

The dielectric tensor for chiral fermions in WSMs has a general structure typical for a magnetized electron-hole plasma:

$$
\epsilon_{i j}=\left(\begin{array}{ccc}
\epsilon_{\perp} & i g & 0 \\
-i g & \epsilon_{\perp} & 0 \\
0 & 0 & \epsilon_{z z}
\end{array}\right)
$$

where $i, j=x, y, z$. However, the expressions for its components and the resulting optical response are far from typical. Consider first the longitudinal component $\epsilon_{z z}=\epsilon_{b}+4 \pi i \sigma_{z z} / \omega$ where $\epsilon_{b}$ is the background dielectric constant of a crystal. The conductivity $\sigma_{z z}$ can be found by calculating the linear response to the longitudinal field $E_{z}=\operatorname{Re}\left[\mathcal{E} e^{i q_{z} z-i \omega t}\right]$. It is convenient to define the optical field through the scalar potential $\phi=\operatorname{Re}\left[\Phi e^{i q_{z} z-i \omega t}\right]$ 
as $\mathcal{E}=-i q_{z} \Phi$. We will assume for simplicity that the Fermi energy for each chirality is between $n=-1$ and $n=1$ and the temperature is low enough so that the states with $n \neq 0$ are either completely filled or empty. The general result for an arbitrary position of the Fermi level is given in [20]. Note also that for the longitudinal field $\boldsymbol{E} \| \boldsymbol{B}$ the transitions between the Landau levels are forbidden in the electric dipole approximation. The resulting linearized density matrix equation for the density matrix elements $\rho_{k k^{\prime}}^{(\chi)}$ for each chirality is

$$
\begin{aligned}
& -i \omega \rho_{k_{z}, k_{z}^{\prime}}^{(\chi)}+i \frac{W_{0}^{(\chi)}\left(k_{z}\right)-W_{0}^{(\chi)}\left(k_{z}^{\prime}\right)}{\hbar} \rho_{k_{z}, k_{z}^{\prime}}^{(\chi)}=-\frac{i}{\hbar} e \Phi \\
& \left\langle n=0, k_{z}\left|e^{i q_{z} z}\right| n=0, k_{z}^{\prime}\right\rangle\left[f_{0}^{(\chi)}\left(k_{z}\right)-f_{0}^{(\chi)}\left(k_{z}^{\prime}\right)\right],
\end{aligned}
$$

where $f_{0}^{(\chi)}\left(k_{z}\right)$ are populations at $n=0$ unperturbed by the optical field and we neglected relaxation, which will be added later. The matrix element in Eq. (5) is calculated using the electron states in a magnetic field [20]; it is equal to the delta-function $\delta_{k_{z}-q_{z}, k_{z}^{\prime}}$. The solution of Eq. (5) in the limit $k_{z} \gg q_{z}$ is

$$
\rho_{k_{z}, k_{z}-q_{z}}^{(\chi)}=\frac{i e \mathcal{E}}{\omega-\chi q_{z} v_{F}} \frac{\partial f_{0}^{(\chi)}\left(k_{z}\right)}{\hbar \partial k_{z}} .
$$

The complex amplitude of the Fourier component of the electric current $j_{z}=\operatorname{Re} \tilde{J} e^{i q_{z} z-i \omega t}$ is given by

$$
\tilde{J}=\sum_{k_{z}, \chi}\left(j_{z}\right)_{k_{z}-q_{z}, k_{z}}^{(\chi)} \rho_{k_{z}, k_{z}-q_{z}}^{(\chi)}
$$

where the matrix element of the spatial Fourier component of the current is

$$
\left(j_{z}\right)_{k_{z}^{\prime}, k_{z}}^{(\chi)}=-e\left\langle n=0, k_{z}^{\prime}\left|e^{-i q_{z} z} \chi v_{F} \sigma_{z}\right| n=0, k_{z}\right\rangle
$$

and the sum can be replaced by integration. The resulting longitudinal component of the conductivity tensor is

$$
\sigma_{z z}=\frac{i e^{3} B v_{F} \omega}{2 \pi^{2} \hbar^{2} c} \frac{1}{\omega^{2}-q_{z}^{2} v_{F}^{2}}
$$

where the $B$-dependence appeared due to the density of states in a quantizing magnetic field. The longitudinal dielectric tensor component therefore takes the form

$$
\epsilon_{z z}=\epsilon_{b}-\omega_{p}^{2} /\left(\omega^{2}-q_{z}^{2} v_{F}^{2}\right)
$$

This result can be also obtained from the kinetic equation [20]. This expression has several peculiar features. First, since the electrons at $n=0$ can move only in one direction with the same velocity $v_{F}$, they cannot bunch in the velocity space and there is no Landau damping. Mathematically, the Landau damping emerges due to contribution from the pole in the integral over electron momenta in the linear conductivity. However, in our case there is no pole in the integral in Eq. (7) since the denominator in Eq. (6) does not depend on the electron momentum.

Second, the effective plasma frequency in Eq. (10) does not depend on the electron density: $\omega_{p}^{2}=\frac{2 \alpha}{\pi} \frac{e B v_{F}}{\hbar}$, where $\alpha=\frac{e^{2}}{\hbar c}$; see also $[9,12]$. In the limit of a uniform electric field $q_{z}=0$ Eq. (9) immediately gives the chiral anomaly. Indeed, if only $n=0$ electrons are involved, the chiral current $j_{\text {chir }}=\partial\left[N^{(\chi=+1)}-N^{(\chi=-1)}\right] / \partial t$ is related to the charge current in a uniform but timedependent field $\boldsymbol{E} \| \boldsymbol{B}$ as $\partial j_{z} / \partial t=-e v_{F} j_{\text {chir }}$. This gives the chiral anomaly current $j_{\text {chir }}=-e^{2} \boldsymbol{E} \boldsymbol{B} /\left(2 \pi^{2} \hbar^{2} c\right)$, in agreement with previous results; see e.g. [2, 3] for review.

The dispersion equation EM waves incident on a magnetized WSM propagate as eigenmodes that can be called magneto-polaritons. They are the solutions of Maxwell's equations for plane waves with the dielectric tensor from Eq. (4). For the photon wave vector $\boldsymbol{q}$ in the $(x z)$-plane making an angle $\theta$ with the magnetic field direction along $z$-axis, they can be written as

$$
\left(\begin{array}{ccc}
\epsilon_{+}-\frac{1}{2} \mu^{2}\left(1+\cos ^{2} \theta\right) & \frac{1}{2} \mu^{2} \sin ^{2} \theta & \frac{1}{\sqrt{2}} \mu^{2} \sin \theta \cos \theta \\
\frac{1}{2} \mu^{2} \sin ^{2} \theta & \epsilon_{-}-\frac{1}{2} \mu^{2}\left(1+\cos ^{2} \theta\right) & \frac{1}{\sqrt{2}} \mu^{2} \sin \theta \cos \theta \\
\frac{1}{\sqrt{2}} \mu^{2} \sin \theta \cos \theta & \frac{1}{\sqrt{2}} \mu^{2} \sin \theta \cos \theta & \epsilon_{z z}-\mu^{2} \sin ^{2} \theta
\end{array}\right)\left(\begin{array}{c}
E_{+} \\
E_{-} \\
E_{z}
\end{array}\right)=0
$$

where $\mu^{2}=\frac{c^{2} q^{2}}{\omega^{2}}, \epsilon_{ \pm}=\epsilon_{\perp} \pm g$, and $E_{ \pm}=\frac{1}{\sqrt{2}}\left(E_{x} \pm i E_{y}\right)$

Longitudinal propagation. For the waves propagating strictly along the magnetic field, i.e. $\theta=0$, the solution to Eqs. (11) consists of two eigenmodes with transverse polarization ("photons"),

$$
\mu_{L, R}^{2}=\epsilon_{ \pm}, \boldsymbol{E}_{\boldsymbol{L}, \boldsymbol{R}}=\frac{1}{\sqrt{2}} E_{ \pm}\left(\boldsymbol{x}_{\mathbf{o}} \pm i \boldsymbol{y}_{\mathbf{o}}\right)
$$

and the wave with the longitudinal polarization $\boldsymbol{E}=$ $E_{z} z_{\mathbf{o}}$ and dispersion equation $\epsilon_{z z}=0$ ("plasmon"). The 
plasmon dispersion is

$$
\omega^{2}=\frac{\omega_{p}^{2}}{\epsilon_{b}}+v_{F}^{2} q^{2} .
$$

We emphasize again that, in contrast to "usual" plasmons, there is no cutoff in Eq. (13) due to Landau damping at large wave vectors $q>\omega / v_{F}$. Therefore, a much stronger plasmon localization is allowed, with propagation only limited by absorption due to scattering on impurities etc.

For oblique propagation, even at very small angles $\theta$, the plasmons and transverse waves are coupled to form hybrid plasmon-polaritons. To determine general trends and obtain analytic results, we neglect the spatial dispersion $\left(q_{z}\right.$-dependence) of $\epsilon_{z z}$ in Eq. (10), which is possible as long as $\mu^{2} \sin ^{2} \theta \ll c^{2} / v_{F}^{2}$. This is not so restrictive since $c / v_{F}>100$. We also neglect any spatial dispersion in $\epsilon_{ \pm}$in the dipole approximation.

It is instructive first to consider the case when the Fermi level is exactly at the Weyl point for both chiralities. In this case, due to electron-hole symmetry the offdiagonal terms in Eq. (4) vanish and the dielectric tensor looks like the one for a uniaxial anisotropic medium. The dispersion equation for the extraordinary wave, i.e. the one polarized in the $(x, z)$ plane, can be written as

$$
\frac{\mu_{x}^{2}}{\epsilon_{z z}}+\frac{\mu_{z}^{2}}{\epsilon_{\perp}}=1
$$

The transverse components of the dielectric tensor are always positive, whereas the $\epsilon_{z z}$ component becomes negative for frequencies below the plasmon resonance, $\omega^{2}<\omega_{p}^{2} / \epsilon_{b}$. In this case Eq. (14) becomes hyperbolic and its isofrequency lines are hyperbolae. Therefore, a magnetized WSM is a natural hyperbolic material at low enough frequencies. Another natural hyperbolic material is hexagonal boron nitride, where the hyperbolic dispersion exists in two narrow spectral ranges near the phonon bands [22]. Otherwise, hyperbolic dispersion is achieved in the effective medium approximation in metal/dielectric metamaterials prepared by nanofabrication [23]. It is promising for numerous applications from superlenses and nanoimaging to photonic integrated circuits. The plasmon resonance frequency $\omega=\omega_{p} / \sqrt{\epsilon_{b}}$ in WSMs which determines the upper bound for hyperbolic dispersion is in the $\mathrm{THz}$ to far-infrared range for a magnetic field of 1-10 Tesla, $\epsilon_{b} \sim 10$ and $v_{F} \sim 10^{8} \mathrm{~cm} / \mathrm{s}$. It is lower than the inter-LL absorption edge for all magnetic fields, so the only loss mechanism is due to scattering on impurities which depends on the material quality.

The ordinary wave in this limit is linearly polarized along $y$-axis and has a standard dispersion $\mu^{2}=\epsilon_{\perp}$.

Fig. 1 shows the dispersion (real part of $\mu$ ) for the extraordinary waves for several different propagation angles $\theta$. Far from inter-LL transitions, we can neglect any dispersion in the transverse part of the dielectric tensor, assuming $\epsilon_{\perp}=\epsilon_{b} \sim 10$. We also added the scattering rate as an imaginary part of frequency $(\omega+i \gamma)$ in the first term of Eq. (5) and took $\gamma$ to be 0.01 of the plasmon resonance frequency $\omega_{\text {res }}=\omega_{p} / \epsilon_{b}^{1 / 2}$. For longitudinal propagation $\theta=0$ the photon dispersion is trivial: $\mu=\sqrt{\epsilon_{b}}$. For any nonzero angle, plasmons and photons hybridize. At the hybrid plasmon-polariton resonance $n$ diverges in the absence of dissipation. The stop band appears between the hybrid resonance and plasmon resonance. It is defined by the condition $\mu^{2}<0$ so that $\operatorname{Re}[\mu]=0$ and the wave cannot propagate. At the boundaries of the stop band $\operatorname{Re}[\mu]$ goes through the value of 1 with a large derivative, leading to a small group velocity $v_{g r} \ll c$. This means that a layer of WSM is able to compress a pulse incident from vacuum by a factor $c / v_{g r}$. All spectral features are tunable by varying the magnetic field or the angle $\theta$.

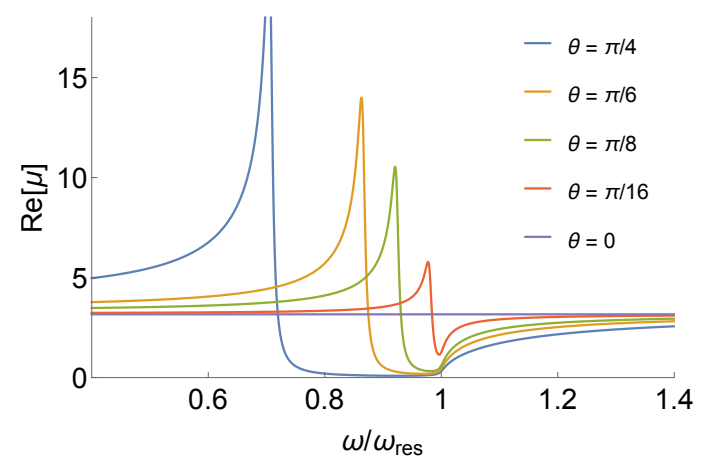

FIG. 1: Dispersion (real part of $\mu(\omega)$ ) of the extraordinary waves in a magnetic field of $10 \mathrm{~T}$ for several different propagation angles $\theta$.

Going back to the general case of an arbitrary Fermi level, Eqs. (11), and arbitrary values of $g$ leads to a biquadratic dispersion equation for $n$ :

$$
\begin{aligned}
& \frac{\mu^{4}}{2}\left(\left(\epsilon_{+}+\epsilon_{-}\right) \sin ^{2} \theta+2 \epsilon_{z z} \cos ^{2} \theta\right)-\left(\epsilon_{+} \epsilon_{-} \sin ^{2} \theta+\right. \\
& \left.\frac{1}{2} \epsilon_{z z}\left(\epsilon_{+}+\epsilon_{-}\right)\left(1+\cos ^{2} \theta\right)\right) \mu^{2}+\epsilon_{+} \epsilon_{-} \epsilon_{z z}=0
\end{aligned}
$$

The polarization coefficients of the normal modes are

$$
\frac{E_{ \pm}}{E_{z}}=\frac{-\frac{1}{\sqrt{2}} \mu^{2} \sin \theta \cos \theta\left(\epsilon_{\mp}-\mu^{2}\right)}{\epsilon_{+} \epsilon_{-}-\frac{1}{2} \mu^{2}\left(1+\cos ^{2} \theta\right)\left(\epsilon_{+}+\epsilon_{-}\right)+\mu^{4} \cos ^{2} \theta} .
$$

Equations (15), (16), and (10) provide a complete description of the electromagnetic wave propagation in WSMs. They can be plotted numerically or solved analytically, leading to cumbersome formulas. In the low temperature limit we obtain analytic expressions for all components of the dielectric tensor, see [20]. Leaving detailed numerical studies to future publications, here we highlight the most interesting cases. Note that a finite separation between Weyl nodes in momentum space by a vector $2 \boldsymbol{b}$ creates an additional anisotropy vector and gives rise to an additional gyrotropic effect $g \propto b[21]$. Therefore, the dispersion shown in Fig. (1) is valid only if this additional $g$ is small. This will be the 
case when the Weyl semimetal is created by applying an external magnetic field to a Dirac semimetal, so that the separation of Weyl points is only due to a Zeeman-type interaction which is typically small. We also note that the expressions for the magnetopolariton dispersion for a nonzero $g$ Eqs. (15) are analytic functions around $g=0$, so the dispersion curves in Fig. (1) will change little when $g$ is small. The most significant effect of a nonzero $g$ is the appearance of an elliptical polarization, Eq. (16) instead of the linear one when $g=0$.

Coupling-induced transparency. For quasilongitudinal propagation, $\sin ^{2} \theta \ll 1$ and plasmonpolariton hybridization occurs in the vicinity of the plasmon resonance, $\left|\epsilon_{z z}\right| \ll 1$. In this case the approximate solution of Eq. (15) is

$$
\begin{aligned}
& \mu_{1,2}^{2}=\frac{1}{\left(\epsilon_{+}+\epsilon_{-}\right) \sin ^{2} \theta+2 \epsilon_{z z}}\left[\epsilon_{+} \epsilon_{-} \sin ^{2} \theta+\epsilon_{z z}\left(\epsilon_{+}+\epsilon_{-}\right)\right. \\
& \left. \pm \sqrt{\left(\epsilon_{+} \epsilon_{-} \sin ^{2} \theta\right)^{2}+\epsilon_{z z}^{2}\left(\epsilon_{+}-\epsilon_{-}\right)^{2}}\right] .
\end{aligned}
$$

The polarization coefficients become

$$
K_{ \pm}=\frac{E_{ \pm}}{E_{z}}=\frac{-\frac{1}{\sqrt{2}} \mu^{2} \sin \theta}{\epsilon_{ \pm}-\mu^{2}} .
$$

In the "non-gyrotropic" limit when $E_{F}=0$ and $\epsilon_{+}=$ $\epsilon_{-}=\epsilon_{\perp}$, the extraordinary wave has dispersion

$$
\mu_{2}^{2}=\frac{\epsilon_{z z} \epsilon_{\perp}}{\epsilon_{\perp} \sin ^{2} \theta+\epsilon_{z z}} ; K_{+}=K_{-}=-\frac{1}{\sqrt{2}} \frac{\epsilon_{z z}}{\epsilon_{\perp} \sin \theta} .
$$

The hybrid resonance corresponds to the vanishing real part of the denominator for $\mu_{2}^{2}$ in Eq. (17) or (19), when $\left|\mu_{2}^{2}\right| \gg 1$.

The effect of coupling-induced transparency emerges near the plasmon resonance where $m u_{2}$ can be of the order of 1 or smaller. When the angle $\theta$ is not too small, $\left|\epsilon_{z z}\right| \ll 1, \sin ^{2} \theta \ll 1$, but $\left|\epsilon_{ \pm}\right| \sin ^{2} \theta \gg\left|\epsilon_{z z}\right|$, the dispersion and polarization of the "extraordinary" wave (the wave that becomes extraordinary if $E_{F}=0$ ) are simply

$$
\mu_{2}^{2}=\frac{\epsilon_{z z}}{\sin ^{2} \theta} ; \frac{E_{x, y}}{E_{z}}=-\frac{\epsilon_{z z}}{2 \sin \theta} \frac{\epsilon_{-} \pm \epsilon_{+}}{\epsilon_{+} \epsilon_{-}} .
$$

In this case one can have $\left|\mu^{2}\right| \ll \epsilon_{ \pm}$whereas the electric field of the wave is directed almost along the magnetic field, i.e. still quasi-longitudinal. Note that $\mu_{2}^{2}$ in Eq. (20) depends only on the $\epsilon_{z z}$ component, which means that the propagation is not affected at all by the resonant inter-LL absorption losses described by the imaginary parts of $\epsilon_{ \pm}$. The medium effectively becomes transparent for this wave! More accurately, its losses are determined only by the imaginary part of $\epsilon_{z z}$, i.e. disorder-related scattering. Within the transparency band, strong plasmon-photon coupling forces the polarization of the wave to be oriented almost along $B$, and therefore it is nearly decoupled from the transitions between LLs. The narrow band of transparency within a broad line of inter-LL absorption is defined by the range of frequencies where $\left|\epsilon_{z z}\right|$ is small enough, namely $\left|\epsilon_{z z}\right| \ll\left|\epsilon_{ \pm}\right| \sin ^{2} \theta$. The situation is similar to the electromagnetically induced transparency (EIT) [24], only in the case of EIT the coupling between two quantum oscillators is provided by a coherent EM drive; see the comparison in [25]. The transparency will be visible if the disorder-related losses determined by $\operatorname{Im} \epsilon_{z z}$ are lower than the inter-Landau level absorption losses determined by $\operatorname{Im} \epsilon_{ \pm}$. Introducing the electron scattering rate $\gamma$ in $\epsilon_{z z}$, one can derive the visibility condition as $\sqrt{\frac{\gamma}{\omega \sin ^{2} \theta}}<\operatorname{Im} \epsilon_{ \pm}$. The value of $\gamma$ depends on the particular material, material quality, and temperature. For example, far-infrared spectroscopy of TaAs [11] (without the magnetic field) found the scattering time varying between 3 and 0.4 ps from low to room temperature. A value of $\operatorname{Im} \epsilon_{+} \sim 3-5$ around $\hbar \omega \sim 100 \mathrm{meV}$ as in Fig. 2 would lead to the visibility condition $\sin \theta>0.01-0.03$.

In the same limit the "ordinary" wave has the dispersion $\mu_{1}^{2}=\frac{2 \epsilon_{+} \epsilon_{-}}{\epsilon_{+}+\epsilon_{-}}$and elliptical polarization in the plane of vectors $\boldsymbol{q}$ and $\boldsymbol{y}_{\mathbf{o}}: \frac{E_{x}}{E_{z}}=\sin \theta$ and $\frac{E_{y}}{E_{z}}=$ $\frac{i\left(\epsilon_{+}+\epsilon_{-}\right)}{\epsilon_{+}-\epsilon_{-}} \sin \theta$.

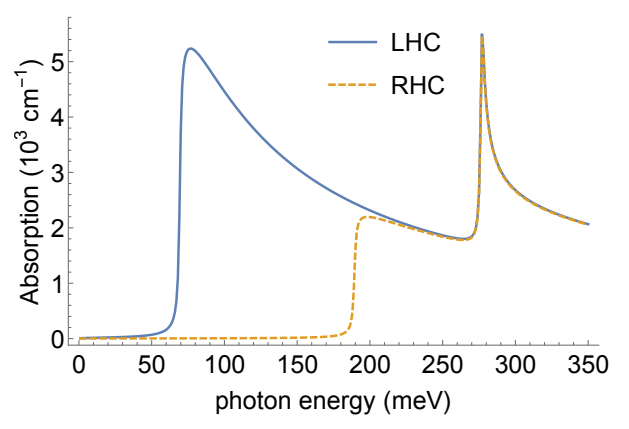

FIG. 2: Absorption spectrum for LHC (solid line) and RHC (dashed line) polarizations in a magnetic field $B=10 \mathrm{~T}$ at zero temperature, the Fermi energy of $60 \mathrm{meV}$, and the relaxation constant $\gamma=1 \mathrm{meV}$.

Intersubband transitions and optical detection of the chiral anomaly. So far we considered peculiar optical properties of WSMs due to massless 1D chiral fermions at the $n=0 \mathrm{LL}$. Here we show that resonant inter-LL absorption from $n=0$ to $n \neq 0$ states provides another sensitive method of studying chiral fermions near Weyl nodes and in particular, detecting the chiral anomaly. Consider the propagation of transverse modes in the Faraday geometry when the eigenmodes are lefthand or right-hand circularly polarized (LHC or RHC). The derivation of the conductivity is outlined in [20]. Fig. 2 gives an example of the absorption spectrum at low temperatures when the Fermi level $E_{F}=60 \mathrm{meV}$ is between $n=0$ and $n=1$ LLs and has the same value for both chiralities. Absorption edges of the lowest-energy transitions $0 \rightarrow 1$, then $-1 \rightarrow 0,-2 \rightarrow 1$, and $-1 \rightarrow 2$ are 
clearly visible in different polarizations (the last two transitions coincide). In particular, there is a broad range of frequencies between 50 and $200 \mathrm{meV}$ when only the LHC polarization is absorbed. Therefore, a several $\mu \mathrm{m}$ thin WSM film can serve as a broadband polarizer converting from linear into circular polarization. Note that both the frequency bandwidth and the polarization coefficient are tunable by a magnetic field and/or Fermi level position. Other obvious applications include optical isolators and saturable absorbers.

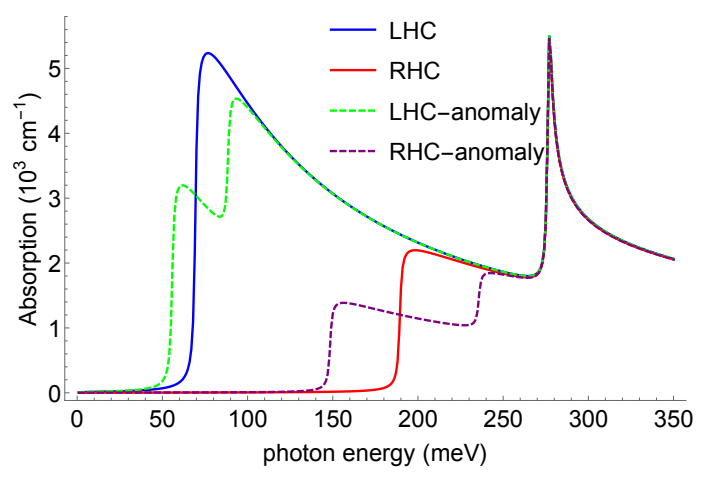

FIG. 3: Absorption spectrum before (solid line) and after (dashed line) a constant electric field $\boldsymbol{E} \| \boldsymbol{B}$ is applied which shifts the Fermi levels by $\pm 30 \mathrm{meV}$ in the two Weyl nodes. The magnetic field is $10 \mathrm{~T}$ and the relaxation constant $\gamma=1$ meV.

Fig. 3 shows the evolution of this spectrum when a constant voltage is applied parallel to the magnetic field, which shifts the Fermi levels for the two chiralities by \pm 30 $\mathrm{meV}$. Here we assumed that before applying bias, the Fermi energy was equal to $60 \mathrm{meV}$ at both Weyl points. As is clear from Fig. 3, when a voltage is applied, an additional absorption edge appears in the spectrum for each polarization, which will be clearly distinguishable as long as the magnitude of the Fermi energy shift is larger than $k_{B} T$. Note that this behavior and the possibility of the optical detection of the chiral anomaly was predicted in Ref. [16].

In conclusion, we showed that unique topological properties of low-energy quasiparticles in WSMs give rise to a plethora of highly unusual magneto-optical effects, which provide an efficient way of studying these fascinating materials and can be utilized in future photonic devices in the terahertz through mid-infrared range. All effects are broadly tunable by varying the magnetic field, electric bias, or the propagation angle. We hope that our study will stimulate further experimental work in this rapidly developing field.

\section{Acknowledgments}

This work has been supported by the Air Force Office for Scientific Research through Grant No. FA9550-15-10153. M.E. and M.T. acknowledge the support by the RFBR grant No. 17-02-00387. A.B. is grateful to Dmitri Kharzeev and Vladimir Zyuzin for useful discussions.
[1] X. Wan, A. M. Turner, A. Vishwanath, and S. Y. Savrasov, Phys. Rev. B 83, 205101 (2011).

[2] P. Hosur and X. Qi, Compt. Rend. Phys. 14, 857 (2013).

[3] O. Vafek and A. Vishwanath, Ann. Rev. Condens. Matter Phys. 5, 83 (2014).

[4] S.-Y. Xu, I. Belopolski, N. Alidoust, M. Neupane, G. Bian, et al., Science 349, 613 (2015).

[5] B. Q. Lv, H. M. Weng, B. B. Fu, X. P. Wang, H. Miao, et al., Phys. Rev. X 5, 031013 (2015).

[6] S.-Y. Xu, N. Alidoust, I. Belopolski, Z. Yuan, G. Bian, et al., Nat. Phys. 11, 748 (2015).

[7] J. Xiong, S. K. Kushwaha, T. Liang, J. W. Krizan, M. Hirschberger, W. Wang, R. J. Cava, and N. P. Ong, Science 350, 413 (2015).

[8] X. Huang, L. Zhao, Y. Long, P. Wang, D. Chen, et al., Phys. Rev. X 5, 031023 (2015).

[9] D. T. Son and B. Z. Spivak, Phys. Rev. B 88, 104412 (2013).

[10] Q. Li, D.E. Kharzeev, C. Zhang, Y. Huang, I. Pletikosic, et al., Nature Phys. 12, 550 (2016).

[11] B. Xu, Y. M. Dai, L. X. Zhao, K. Wang, R. Yang, et al., Phys. Rev. B 93, 121110(R) (2016).

[12] B. Z. Spivak and A. V. Andreev, Phys. Rev. B 93, 085107 (2016).

[13] J. Zhou, H. Chang, and D. Xiao, Phys. Rev. B 91, 035114
(2015).

[14] F.M.D. Pellegrino, M. I. Katsnelson, and M. Polini, Phys. Rev. B 92, 201407(R) (2015).

[15] C. J. Tabert, J. P. Carbotte, and E. J. Nicol, Phys. Rev. B 93, 085426 (2016).

[16] P.E.C. Ashby and J. P. Carbotte, Phys. Rev. B 89, 245121 (2014).

[17] J. Ma and D. A. Pesin, Phys. Rev. B 92, 235205 (2015).

[18] E.V. Gorbar, V.A. Miransky, I.A. Shovkovy, and P.O. Sukachov, Phys. Rev. B 95, 115202 (2017).

[19] P.E.C. Ashby and J. P. Carbotte, Phys. Rev. B.87, 245131 (2013).

[20] Supplemental Material, available online.

[21] A.A. Zyuzin and A.A. Burkov, Phys. Rev. B 86, 115133 (2012).

[22] S. Dai, Q. Ma, M. K. Liu, T. Andersen, Z. Fei, et al., Nat. Nanotech. 10, 682 (2015).

[23] A. Poddubny, I. Iorsh, P. Belov, and Y. Kivshar, Nat. Phot. 7, 958 (2013).

[24] M. Fleischhauer, A. Imamoglu, and J. P. Marangos, Rev. Mod. Phys. 77, 633 (2005).

[25] M. D. Tokman, M. A. Erukhimova, Radiophysics and Quantum Electronics, vol. 57, 821-836 (2015). 